\title{
An Exploratory Study of the Use of the Electronic Health Records of Hypertensive Patients to Support the Primary Prevention of Stroke in Shanghai
}

This article was published in the following Dove Press journal:

Risk Management and Healthcare Policy

\section{Tingting Yang ${ }^{1} *$ \\ Fen $\mathrm{Li}^{2}$,* \\ Bifan Zhu (iD ${ }^{2}$ \\ Yuqian Chen ${ }^{2}$ \\ Duo Chen ${ }^{2}$ \\ Changying Wang ${ }^{2}$ \\ Zhiying Hou ${ }^{2}$ \\ Jiajie $X u\left(D^{2}\right.$ \\ Shuwei $\mathrm{Gu}^{3}$ \\ Jiefeng Liu (iD ${ }^{4}$ \\ Zhuochun $\mathrm{Wu}^{\prime}$ \\ Ying Wang' \\ Chunlin Jin ${ }^{2}$}

'School of Public Health/ Key Laboratory of Health Technology Assessment,

National Health and Family Planning

Commission of the People's Republic of

China, Fudan University, Shanghai,

People's Republic of China; ${ }^{2}$ Shanghai

Health Development Research Center,

Shanghai Medical Information Center,

Shanghai, People's Republic of China;

${ }^{3}$ Jiangxi University of Traditional Chinese

Medicine, School of Economics and

Management, Nanchang, Jiangxi, People's

Republic of China; ${ }^{4}$ Department of

Epidemiology and Health Statistics,

Xiangya School of Public Health, Central

South University, Changsha, People's

Republic of China

*These authors contributed equally to this work

Correspondence: Chunlin Jin; Ying Wang Email jinchunlin@shdrc.org;

wangying1013@fudan.edu.cn
Background: The value of identifying and targeting population demographics at high risk of stroke based on patient-reported outcomes (PROs) with electronic health records (EHRs) in Shanghai is largely undiscovered.

Aim: To test the hypothesis that establishing an evidence-based support system composed of PROs integrated with EHRs could be effective at identifying individuals at high risk of suffering from stroke.

Methods: The patients included in this study joined the hypertensive patient management system from 2014 to 2018. We merged the Hypertension Patients Management Database and the Diabetes Mellitus Patients Management Database of Shanghai Jiading district, then kept the hypertension patients with or without diabetes. We subsequently performed a screen analysis utilizing EHRs to target the population with any risk factor for stroke, namely, hypertension, diabetes mellitus, obesity, smoking and physical inactivity. We also calculated the distribution of each risk factor and the combinations of risk factors.

Results: In the Jiading District of Shanghai, 46,580 hypertensive patients with complete baseline information joined the hypertensive patient management system from 2014 to 2018 . The majority of the patients were aged above 60 years old. Physical inactivity $(83.24 \%)$, smoking $(24.07 \%)$, diabetes $(16.87 \%)$, and obesity $(12.23 \%)$ were highly prevalent in hypertensive participants. Approximately 4377 patients were diagnosed with hypertension exclusively, accounting for $9.70 \%$ of the total number of patients in this study. Meanwhile, approximately $52.47 \%$ of the patients were diagnosed with two concurrent risk factors, and $38.13 \%$ of the patients had hypertension, meaning that 17,762 patients could be labeled as the high-risk population for stroke according to the criteria established by the National Stroke Screening Survey.

Conclusion: Our exploratory findings demonstrate the feasibility of pinpointing and targeting populations at high risk of stroke using the EHRs of hypertensive patients.

Keywords: hypertension, electronic health records, EHRs, stroke risk factors

\section{Background}

With over 2 million new cases annually, stroke is associated with the highest disabilityadjusted life years lost of any disease in China. ${ }^{1}$ The prevalence of stroke is expected to increase even further as a result of population aging, an ongoing high prevalence of risk factors (such as hypertension), and inadequate medical management. ${ }^{1}$ Stroke is 
the second-leading cause of death worldwide and the leading cause of death in China. ${ }^{2,3}$ Up to $80 \%$ of strokes can be prevented through healthy lifestyle changes and proper management of health conditions that raise the risk of stroke. ${ }^{4}$

From 2013 to 2014, the National Project Office of Stroke Prevention and Control organized a screening survey of 633,859 residents aged above 40 years old across 31 Chinese provinces. ${ }^{1}$ Following a random cluster sampling framework, structured questionnaires were used to collect information on risk factors, medical history, treatments received, and sociodemographic characteristics, administered by community health workers trained by national and provincial specialists. ${ }^{5}$ Hypertension, dyslipidaemia, diabetes, obesity, physical inactivity, smoking, and atrial fibrillation are the most common and modifiable risk factors for stroke in China. ${ }^{6,7}$

In China, the traditional way of identifying demographics at high risk of stroke is using questionnaires to gather information from certain population groups, and the sample size can vary in different research settings. ${ }^{1,8,9}$ Usually, a largescale survey requires a substantial amount of time and manpower. We are currently living in a fast-changing world of information and communications technology. A patientreported outcome (PRO) is a measurement of any aspect of a patient's health status that comes directly from the patient. ${ }^{10}$ PROs are used for risk factors identification of some diseases, such as metabolic syndrome, ${ }^{11}$ cardiac disease, ${ }^{12}$ hip impairment, ${ }^{13}$ mental distress, ${ }^{14}$ systemic sclerosis. ${ }^{15}$ As a result of the continuous creation of electronic health record databases, local EHRs data including PROs can now allow researchers to capture, aggregate, access, and analyse more data than ever before, including new and miscellaneous sources of data, such as data on hypertension, diabetes, overweight or obesity.

Being extensively used in western countries, EHRs have also been adopted by most hospitals in China, especially tertiary hospitals. ${ }^{16,17}$ The nationwide survey shows that China's health informatization is rapidly growing. However, the potential of converged data is still far from being fully exploited and many more data applications are still under development, with low data quality and lack of uploading detailed clinical information in some systems. ${ }^{16,18}$ Despite these limitations, real-world evidence-based stroke prevention should still be given more attention. A regional model of screening a high-risk stroke population supported by an EHR database in China is needed, which would help to investigate the distribution of stroke risk factors, identify more populations at high risk of stroke, and develop a public health action plan for stroke prevention.

Jiading is a suburban district located in the northwest region of Shanghai. Back in 2018, it had a population of 639.3 thousand people, with $33.47 \%$ of the population aged above 59 years old. ${ }^{19}$ Hypertension, diabetes mellitus, smoking, obesity, and physical inactivity are investigated in the Stroke Prevention and Control Project in China as stroke risk factors. Additionally, hypertension is a particularly important risk factor for haemorrhagic stroke, although it contributes to atherosclerotic disease, which can lead to ischaemic stroke as well. ${ }^{20}$ Thus, we conducted an exploratory study of stroke risk factors analysis based on the hypertensive patient management system in Jiading, which aimed to estimate the distribution of the major risk factors for stroke prior to a formal survey or door-to-door investigation. Using electronic health records may enable to perform comprehensive data analysis and provide customized recommendations for the primary prevention of stroke.

\section{Methods Database Source}

Two databases including information on topics such as hypertension, diabetes, smoking, physical activity, height, and weight were selected for analysis in this study: the Hypertension Patients Management Database and the Diabetes Mellitus Patients Management Database. Both databases cover the Shanghai Jiading District household registration population only. The Hypertension Patients Management Database documents clinical information on hypertensive patients' height, weight, smoking history, alcohol consumption, physical activity, systolic blood pressure, diastolic blood pressure and medications. Whereas the Diabetes Mellitus Patients Management Database is used to record information on the type of diabetes mellitus, systolic blood pressure, diastolic blood pressure and fasting blood sugar value.

\section{Patient Enrollment}

The registry recruited patients in Jiading District from 2014 to 2018 who met the following criteria:

- Older than 40 years old.

- A diagnosis of hypertension with or without diabetes.

- Shanghai Jiading District household registration population. 


\section{Exclusion Criteria}

- Died before 2019.

- A past history of stroke.

- Lack of baseline data.

\section{Data Extraction}

We used a standardized data collection form (available on request). All patients from the Hypertension Patients Management Database had a confirmed diagnosis of hypertension, and all patients from the Diabetes Mellitus Patients Management Database were diagnosed with diabetes mellitus. After merging these two databases into a data source, we retained the hypertensive patients with or without diabetes for analysis. We extracted the identity card number and information on sex, age, height, weight, smoking, alcohol consumption, physical activity, and diabetes. Only the data from patients aged 40 years old or more were processed. The patients joined the hypertensive patient management system from 2014 to 2018 .

\section{Data Synthesis and Analysis}

In this article, the analyses focused on characterizing five risk factors for stroke, namely, hypertension, diabetes, obesity, smoking and physical inactivity. We followed the China National Stroke Screening Survey's standard criteria for hypertension, diabetes, obesity, and smoking. ${ }^{21} \mathrm{RF} 1$ : Hypertension, defined as a history of high blood pressure $(\geq 140 / 90 \mathrm{mmHg}$ ) reported by the participant or a diagnosis of hypertension made by physicians from a tier 2 hospital or above. RF2: Diabetes mellitus defined by previous diagnosis, or a fasting plasma glucose level $\geq 7.0 \mathrm{mmol} / \mathrm{L}$ or a random blood glucose level $\geq 11.0 \mathrm{mmol} / \mathrm{L}$. RF3: Obesity, characterized by the body mass index (BMI), calculated as the patient's weight in kilograms divided by the square of the patient's height in metres. Based on the WHO's definition of obesity, obesity is defined as a BMI $\geq 30 \mathrm{~kg} / \mathrm{m}^{2}$, overweight is considered as a BMI of $25-30 \mathrm{~kg} /$ $\mathrm{m}^{2.22}$ According to China's standards on weight management for overweight or obese people, the BMI was categorized into four groups in our study: Underweight (BMI $\left.<18.5 \mathrm{~kg} / \mathrm{m}^{2}\right)$, normal weight $\left(18.5 \mathrm{~kg} / \mathrm{m}^{2} \leq\right.$ BMI $<24 \mathrm{~kg} /$ $\mathrm{m}^{2}$ ), overweight $\left(24 \mathrm{~kg} / \mathrm{m}^{2} \leq \mathrm{BMI}<28 \mathrm{~kg} / \mathrm{m}^{2}\right)$, and obesity (BMI $\geq 28 \mathrm{~kg} / \mathrm{m}^{2}$ ). ${ }^{23} \mathrm{RF} 4$ : Smoking, defined as smoking any tobacco product continuously or cumulatively for more than 6 months during the patient's lifetime. RF5: Regarding physical inactivity, we considered exercising on a daily basis as physical activity, and the other categories were classified as physical inactivity. Proportions were used for categorical variables, and continuous variables were expressed in the form of mean $\pm \mathrm{SD}$ (Standard deviation).

\section{Statistical methods}

Descriptive statistics were calculated for all variables. Continuous data were presented as mean $\pm \mathrm{SD}$ and compared using the Student's $t$-test. Categorical variables were displayed as frequencies and percentages and analysed using chi-square $\left(\chi^{2}\right)$ tests. A two-tailed $p<0.05$ was considered statistically significant. All analyses were conducted using the Stata 13.0 software.

\section{Results}

In this exploratory study, we selected two databases for research. We initially screened 49,516 people included into the hypertensive patient management system from 2014 to 2018, out of which 2936 individuals were eliminated based on our inclusion and exclusion criteria. The detailed patient recruitment flow chart is illustrated in Figure 1. Overall, this study enrolled a total of 46,580 patients with at least one risk factor for stroke who had complete baseline information.

An introduction of the database is summarized in Table 1; on an annual basis from 2014 to 2018, 10, 595, 20,077, 12,876, and 3032 patients were entered into the system, respectively. A detailed description of the participants' follow-up duration is presented in Figure 2 for hypertensive patients.

Patient demographic characteristics are listed in Table 2. The proportion of males was $48.43 \%$, while $51.57 \%$ of these patients were females. The majority of the patients (79.12\%) were older than 60 years old. The mean age was 67.82 years old. Approximately half of the patients (42.49\%) had normal BMIs. Compared with women, men had a higher BMI (24.69 vs $24.48 \mathrm{~kg} / \mathrm{m}^{2}$ ), smoking rate ( $48.90 \%$ vs $0.76 \%)$, alcohol consumption rate $(46.44 \%$ vs 2.07\%), SBP (130.60 vs $130.07 \mathrm{~mm} \mathrm{Hg}$ ), and DBP (79.69 vs $79.04 \mathrm{~mm} \mathrm{Hg})($ all $\mathrm{p}<0.05)$.

Table 3 shows the variation of risk factors with respect to gender and age. Compared with men, women were more likely to have obesity ( $<<0.05$ ), diabetes and physical inactivity. However, no significant gender differences were detected for diabetes or physical inactivity $(\mathrm{p}>0.05)$. Men were far more likely to consume alcohol and smoke than women $(\mathrm{p}<0.05)$. The rate of diabetes among hypertensive patients aged 70-79 years old was higher 


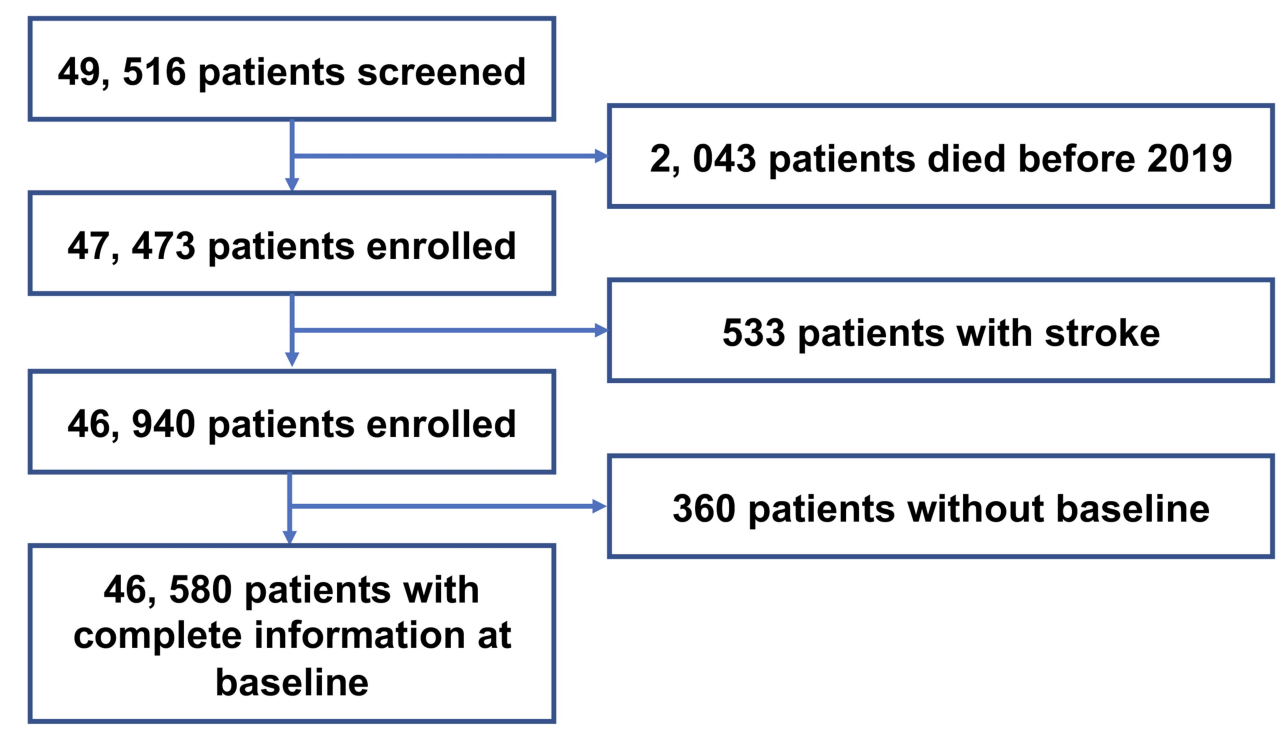

Figure I Flowchart of inclusion data in the present study.

than that among patients in other age groups. Moreover, the prevalence of smoking, obesity and physical inactivity among hypertensive patients aged 40-49 years old was higher than that among patients in other age groups. The proportion of patients with diabetes, smoking, obesity and physical inactivity differed among age groups (all $\mathrm{p}<0.05$ ).

Approximately $9.40 \%$ of patients had hypertension only. Compared with women, men were less likely to have only one risk factor $(7.03 \%$ vs $11.62 \%)$ and two risk factors $(38.59 \%$ vs $65.51 \%)$ for stroke; nonetheless, men had a higher rate of carrying three risk factors $(42.67 \%$ vs $20.06 \%$ ), four risk factors (10.58\% vs $2.79 \%)$, and five risk factors $(1.13 \%$ vs $0.01 \%)$ (Figure 3 ). The risk factors distribution between males and females was different, possibly due to the fact that only a few women smoked.

For men, most patients fell within the category of hypertension + smoking + physical inactivity $(31.35 \%)$, followed by hypertension + physical inactivity $(31.19 \%)$ and hypertension only $(7.03 \%)$. For women, were mostly identified within the category of hypertension + physical inactivity (61.08\%), followed by hypertension only

Table I Number of Hypertension Patients Newly Entered into the Present EHR Database Annually

\begin{tabular}{|l|l|l|l|l|l|}
\hline Gender & $\mathbf{2 0 1 4}$ & $\mathbf{2 0 1 5}$ & $\mathbf{2 0 1 6}$ & $\mathbf{2 0 1 7}$ & $\mathbf{2 0 1 8}$ \\
\hline Male & 5180 & 9809 & 5972 & 1597 & 22,558 \\
Female & 5415 & 10,268 & 6904 & 1435 & 24,022 \\
Total & 10,595 & 20,077 & 12,876 & 3032 & 46,580 \\
\hline
\end{tabular}

$(11.62 \%)$ and hypertension + diabetes + physical inactivity (11.06\%) (Figure 4).

\section{Discussion}

\section{Main Findings}

Our study highlighted the astonishing occurrence rate of several modifiable stroke risk factors in hypertensive patients via EHRs in Shanghai based on a large contemporary population. The increasing burden of stroke has become a serious public health issue and an enormous economic burden in Shanghai. Consequently, strategies focusing on data analysis should be emphasized in the available health monitoring data area before the qualified primary prevention of stroke is addressed.

The data contained in EHRs could be used for recognition of risk factors, developing and validating a cardiovascular risk score, predicting stroke events and assisting physicians to provide prevention measures for patients or individuals at high risk. For example, a stroke phenotype risk factor was extracted from the Veteran Health Administration clinical reports. ${ }^{24}$ The cardiovascular health $(\mathrm{CVH})$ score within electronic health records was used to generate visualizations in a study; a CVH score comprised information on the smoking status, body mass index, blood pressure, cholesterol, fasting glucose, physical activity, and diet, and aimed to improve healthcare providers' practice by rendering these personalized EHR data available at the point-of-care for prevention discussions with patients. ${ }^{25}$ Similarly, a model with an outcome of myocardial infarction, stroke or CVD death 


\section{0,000}

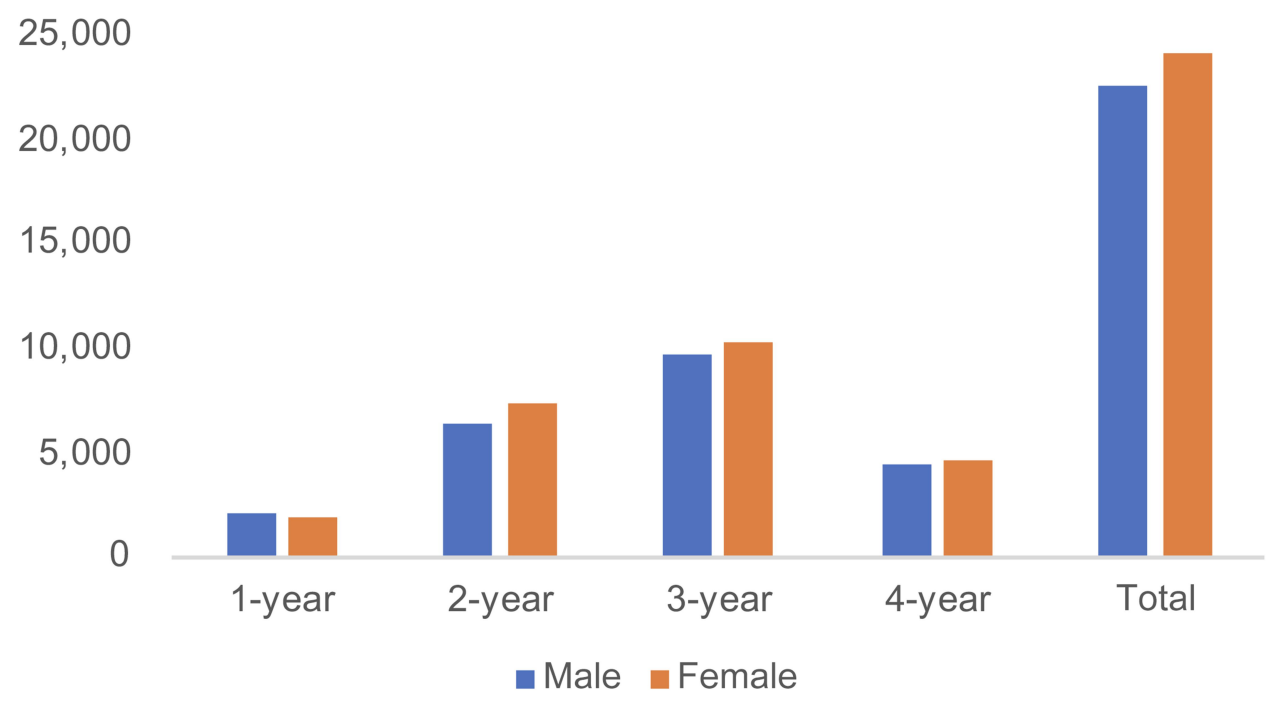

Figure 2 Number of hypertensive patients in the present EHR database by years of follow-up.

within 2 years was developed in The New Zealand (NZ) PREDICT CVD Cohort Study. ${ }^{26}$

Hypertension has been documented as the most important risk factor for stroke in China. ${ }^{27}$ For that reason, in our study design, all of the subjects had a confirmed diagnosis of hypertension. The second-most common risk factor in our study population was physical inactivity; the rate of physical inactivity was quite high because the

Table 2 Patient Demographic Characteristics

\begin{tabular}{|c|c|c|c|c|c|}
\hline Characteristic & Overall & Male & Female & $\mathbf{t}$ & p value \\
\hline Participant, n (\%) & 46,580 & $22,558(48.43)$ & $24,022(51.57)$ & & \\
\hline Age (year) & $67.82 \pm 10.59$ & $66.80 \pm 10.52$ & $68.78 \pm 10.58$ & -20.191 & 0.000 \\
\hline Age group, n (\%) & & & & & 0.000 \\
\hline $40-49$ & I8II (3.89) & $1185(5.25)$ & $626(2.6 \mathrm{I})$ & & \\
\hline $50-59$ & 7916 (16.99) & $4 I I I(18.22)$ & $3805(15.84)$ & & \\
\hline $60-69$ & $18,132(38.93)$ & $8846(39.21)$ & $9286(38.66)$ & & \\
\hline $70-79$ & $11,516(24.72)$ & $5530(24.51)$ & $5986(24.92)$ & & \\
\hline$\geq 80$ & 7205 (15.47) & $2886(12.79)$ & $4319(17.98)$ & & \\
\hline BMI (kg/m2) & $24.58 \pm 3.13$ & $24.69 \pm 2.92$ & $24.48 \pm 3.31$ & 7.200 & 0.000 \\
\hline BMI group, n (\%) & & & & & 0.000 \\
\hline Underweight $<18.5$ kg/m2 & $766(1.64)$ & $281(1.25)$ & $485(2.02)$ & & \\
\hline Normal weight $18.5 \sim 23.9 \mathrm{~kg} / \mathrm{m} 2$ & $|9,79|(42.49)$ & $9153(40.58)$ & $10,638(44.28)$ & & \\
\hline Overweight $24 \sim 27.9$ kg/m2 & $20,324(43.63)$ & $10,533(46.69)$ & 9791 (40.76) & & \\
\hline Obesity $\geq 28 \mathrm{~kg} / \mathrm{m} 2$ & $5699(12.23)$ & 2591 (II.49) & $3108(12.94)$ & & \\
\hline Smoking, n (\%) & $11,212(24.07)$ & $|I, 03|(48.9)$ & $18 \mid(0.76)$ & & 0.000 \\
\hline Alcohol consumption, n (\%) & $10,974(23.56)$ & $10,476(46.44)$ & $498(2.07)$ & & 0.000 \\
\hline \multicolumn{6}{|l|}{ Blood pressure $(\mathrm{mm} \mathrm{Hg})$} \\
\hline SBP & $130.33 \pm 9.45$ & $130.60 \pm 9.44$ & $130.07 \pm 9.44$ & 5.997 & 0.000 \\
\hline DBP & $79.35 \pm 6.24$ & $79.69 \pm 6.31$ & $79.04 \pm 6.16$ & 11.252 & 0.000 \\
\hline
\end{tabular}

Notes: P-values resulted from two independent samples from the $t$-test and chi-square test for continuous and categorical variables, respectively. Values were expressed as the mean (SD) and frequency (percentage) for continuous and categorical variables, respectively. 
Table 3 Four Risk Factors of Stroke in Hypertensive Patients in the Present Study

\begin{tabular}{|c|c|c|c|c|}
\hline & Diabetes (\%) & Smoking (\%) & Obesity (\%) & Physical Inactivity (\%) \\
\hline Total & $7856(16.87)$ & II,2I 2 (24.07) & $5699(12.23)$ & $38,772(83.24)$ \\
\hline \multicolumn{5}{|l|}{ Gender } \\
\hline Male & $3788(16.79)$ & $|I, 03|(48.9)$ & 2591 (II.49) & $18,728(83.02)$ \\
\hline Female & $4068(16.93)$ & $|8|(0.75)$ & $3108(12.94)$ & $20,044(83.44)$ \\
\hline $\mathrm{P}$ & 0.682 & 0.000 & 0.000 & 0.227 \\
\hline \multicolumn{5}{|l|}{ Age group } \\
\hline $40-49$ & $136(7.51)$ & $685(37.82)$ & $303(16.73)$ & $1618(89.34)$ \\
\hline $50-59$ & $1050(13.26)$ & $2685(33.92)$ & $1070(13.52)$ & 6799 (85.89) \\
\hline $60-69$ & $3191(17.6)$ & $4908(27.07)$ & $2313(12.76)$ & $\mid 4,780(8|.5|)$ \\
\hline $70-79$ & $2347(20.38)$ & $2205(19.15)$ & $1362(11.83)$ & 9307 (80.82) \\
\hline$\geq 80$ & $1132(15.71)$ & $729(10.12)$ & $651(9.04)$ & $6268(87.00)$ \\
\hline $\mathrm{P}$ & 0.000 & 0.000 & 0.000 & 0.000 \\
\hline
\end{tabular}

Notes: P-values resulted from the chi-square test for categorical variables. Values are presented as frequency (percentage) for categorical variables.

categories of physical activity from the original data were exercising everyday, exercising one or more days per week, exercising once per month, and not exercising.

\section{A}

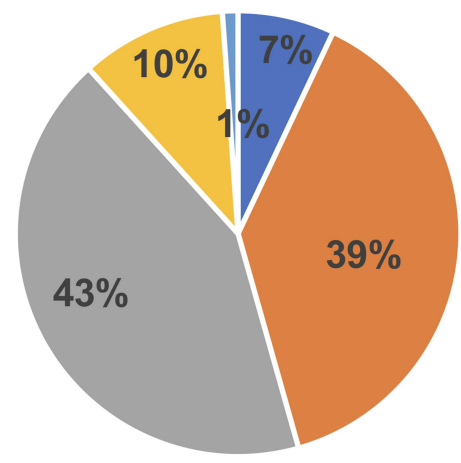

- One risk factor " Two risk factors " Three risk factors
" Four risk factors " Five risk factors

B

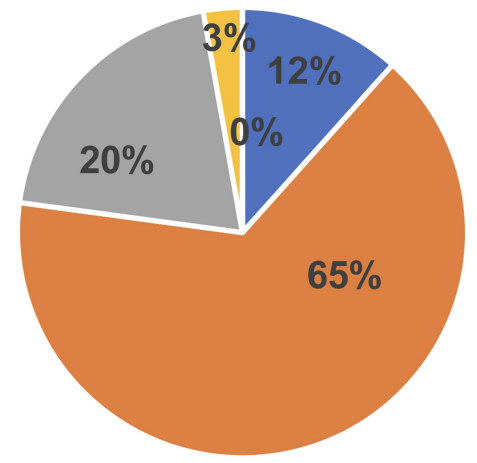

\section{- One risk factor - Two risk factors - Three risk factors \\ - Four risk factors . Five risk factors}

Figure 3 (A) Percentage of number of risk factors distribution in male patients. (B) Percentage of number of risk factors distribution in female patients.
The definition of physical inactivity from the China National Stroke Screening Survey was less than 3 times of physical activity for 30 minutes per week. Thus, the rate of physical inactivity may have been overestimated in our study. One large study revealed a 50.01\% survival among stroke patients with physical inactivity. ${ }^{28}$

Concerning the analysis based on gender, this study found that women were more likely to have diabetes and obesity than men. Similar results were found in a study of stroke patients aged 60 years old or above in northern China $^{11}$ and in another study of stroke patients aged 75 years old or above in China. ${ }^{29}$ In this study, alcohol abuse and smoking were more common among males, which is consistent with several previous findings. ${ }^{11,30}$

We also found that the risk factors among hypertension patients varied by age. A higher proportion of hypertension patients aged 70-79 years old had concurrent diabetes mellitus. The rates of smoking, obesity and physical inactivity among hypertensive patients aged 40-49 years old were higher than the rates in other age groups, but the number of patients aged 40-49 years old was lower. This could be attributed to an uneven age group distribution in Shanghai Jiading District. Another possible explanation is that compared with their parents' generation, patients aged 40-49 years old may lead completely different lifestyles with more energy intake and less physical activity, thus increasing their probability of carrying risk factors for obesity.

The China National Stroke Screening Survey defined people with a high risk of stroke as those with transient ischemic attack (TIA), stroke, or three risk factors (RFs: hypertension, atrial fibrillation or valvular heart disease, 
Hypertension+diabetes+smoking+obesity+physical inactivity

Hypertension+diabetes+obesity+physical inactivity

Hypertension+smoking+obesity+physical inactivity

Hypertension+diabetes+smoking+physical inactivity

Hypertension+diabetes+smoking+obesity

Hypertension+obesity+physical inactivity

Hypertension+smoking+physical inactivity

Hypertension+smoking+obesity

Hypertension+diabetes+physical inactivity

Hypertension+diabetes+obesity

Hypertension+diabetes+smoking

Hypertension+physical inactivity

Hypertension+obesity

Hypertension+smoking

Hypertension+diabetes

Hypertension$$
0
$$

-Female $\square$ Male

Figure 4 Combinations of stroke risk factors in the present study.

smoking, dyslipidaemia, diabetes, physical inactivity, obesity, family history of stroke). ${ }^{21}$

In our research, targeting hypertensive patients who did or did not smoke and who had or did not have diabetes, physical inactivity, and obesity was rather simple. We noticed a high rate of those risk factors in hypertension populations in the present study, and poor management of these risk factors may be conducive to the high prevalence of stroke. Compared with other traditional populationbased surveys, ${ }^{1,9}$ this study provides a comprehensive and accurate estimation of the rate of risk factors with a more representative population. The primary prevention and management of related risk factors based on insights from EHR data might be of tremendous importance.

\section{Limitations}

Several potential limitations of this study should be noted. First, none of the two databases included all the patients with hypertension or diabetes in Jiading District, which implies that the number of people at high risk of stroke in our study might be underestimated. Second, the structural database was designed before the China National Stroke Screening Survey 2016 was initiated, and information on the history of TIA, atrial fibrillation or valvular heart disease, dyslipidaemia, family history of stroke was not included or was inconsistent with the survey details. Third, we considered exercising six

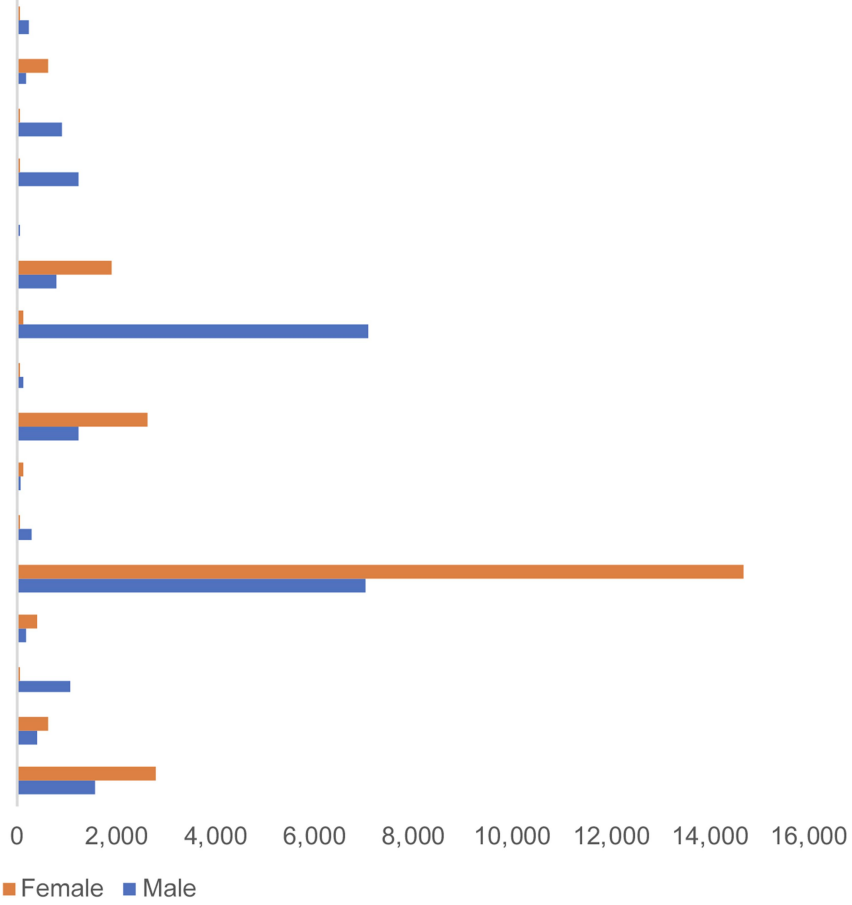

days per week or less as physical inactivity, meanwhile the definition of physical inactivity from the China National Stroke Screening Survey was physical activity performed less than 3 times a week for 30 minutes. Hence, we may have overestimated the rate of physical inactivity.

\section{Conclusions}

We hereby provided a large population and accurate estimation of the current prevalence rate of stroke risk factors in Shanghai's Jiading District hypertensive patients. Patients with three or more risk factors accounted for $38.13 \%$ of the sample, which means that 17,762 patients could be identified as the high-risk population for stroke according to the criteria established by the National Stroke Screening Survey. These patients may be the source of great economic and social burdens. Furthermore, the considerable rate of stroke risk factors we detected was likely related to the poor management of pre-existing conditions and unhealthy lifestyle choices, particularly smoking and physical inactivity. Therefore, strategies of long-term data mining from EHRs could contribute to the primary prevention of stroke in Shanghai.

\section{Data Sharing Statement}

The datasets used and/or analyzed during the current study are available from the corresponding author on reasonable request. 


\section{Ethics Approval and Consent to Participate}

Ethical clearance was obtained from Shanghai Health Development Research Center, Shanghai Medical Information Center. Neither the case records nor the data extracted was used for any other purpose. All data accessed complied with relevant data protection and privacy regulations.

\section{Acknowledgments}

We thank JiaDing Health Information Center for supporting the study. We are grateful to staff members of JiaDing Health Information Center, data collectors and study participants for their cooperation in the success of this study.

\section{Author Contributions}

All authors made a significant contribution to the work reported, whether that is in the conception, study design, execution, acquisition of data, analysis and interpretation, or in all these areas; took part in drafting, revising or critically reviewing the article; gave final approval of the version to be published; have agreed on the journal to which the article has been submitted; and agree to be accountable for all aspects of the work.

\section{Funding}

This work was support by three grants, Shanghai Municipal Health Commission 2018YQ51, the National Natural Science Foundation of China (71673055), Shanghai Municipal Science and Technology Major Project (No.2018SHZDZX01), and ZJLab. The funder had no role in study design, data collection and analysis, decision to publish or preparation of the manuscript.

\section{Disclosure}

The authors declare that they have no competing interests for this work.

\section{References}

1. Wu S, Wu B, Liu M, et al. Stroke in China: advances and challenges in epidemiology, prevention, and management. Lancet Neurol. 2019;18 (4):394-405. doi:10.1016/S1474-4422(18)30500-3

2. GBD 2016 Causes of Death Collaborators. Global, regional, and national age-sex specific mortality for 264 causes of death, 1980-2016: a systematic analysis for the Global Burden of Disease Study 2016. Lancet. 2017;390:1151-1210. doi:10.1016/S01406736(17)32152-9

3. Zhou M, Wang H, Zhu J, et al. Cause-specific mortality for 240 causes in China during 1990-2013: a systematic subnational analysis for the Global Burden of Disease Study 2013. Lancet. 2016;387 (10015):251-272. doi:10.1016/S0140-6736(15)00551-6
4. Prevention U S C F. Preventing Stroke: healthy Living. Available from: https://www.cdc.gov/stroke/healthy_living.htm. Accessed September 8, 2020.

5. Li J, Wang L, Chao B, et al. Prevalence of stroke in China: an epidemiological study based on the National Stroke Screening Survey. Lancet. 2015;386:S49. doi:10.1016/S0140-6736(15)00630-3

6. Wang W, Jiang B, Sun H, et al. Prevalence, incidence, and mortality of stroke in China: results from a nationwide population-based survey of 480687 adults. Circulation. 2017;135:759-771. doi:10.1161/ CIRCULATIONAHA.116.025250

7. Guan T, Ma J, Li M, et al. Rapid transitions in the epidemiology of stroke and its risk factors in China from 2002 to 2013. Neurology. 2017;89(1):53-61. doi:10.1212/WNL.0000000000004056

8. Wang Y, Wang J, Cheng J, Liang X, Li X, Lu W. Is the population detected by screening in China truly at high risk of stroke? J Stroke Cerebrovasc Dis. 2018;27(8):2118-2123. doi:10.1016/j.jstrokecere brovasdis.2018.03.009

9. Mi T, Sun S, Zhang G, et al. Relationship between dyslipidemia and carotid plaques in a high-stroke-risk population in Shandong Province, China. Brain Behav. 2016;6(6):e473. doi:10.1002/brb3.473

10. U.S. Department of Health and Human Services FDA Center for drug evaluation and Research, U.S Department of Health and Human Services FDA Center for Biologics Evaluation and Research, U.S Department of Health and Human Services FDA Center for Devices and Radiological Health. Guidance for Industry: Patient-reported Outcome Measures: Use in Medical Product Development to Support Labeling Claims: Draft Guidance. Health Qual Life Outcomes; 2006.

11. Lin CH, Chiang SL, Tzeng WC, Chiang LC. Systematic review of impact of lifestyle-modification programs on metabolic risks and patient-reported outcomes in adults with metabolic syndrome. Worldviews Evid Based Nurs. 2014;11:361-368. doi:10.1111/ wvn. 12069

12. Berg SK, Rasmussen TB, Thrysoee L, et al. Mental health is a risk factor for poor outcomes in cardiac patients: findings from the national DenHeart survey. $J$ Psychosom Res. 2018;112:66-72. doi:10.1016/j.jpsychores.2018.07.002

13. Schafer KA, Clohisy JC, Nepple JJ. Rapidly progressive arthritis in femoroacetabular impingement: patient characteristics and risk factors for total hip arthroplasty by the age of forty. Iowa Orthop $J$. 2020;40:129-134.

14. Tang L, Zhang Y, Pang Y. Patient-reported outcomes from the distress assessment and response tool program in Chinese cancer inpatients. Psychooncology. 2020;29:869-877. doi:10.1002/pon.5358

15. Ingegnoli F, Ughi N, Mihai C. Update on the epidemiology, risk factors, and disease outcomes of systemic sclerosis. Best Pract Res Clin Rheumatol. 2018;32(2):223-240. doi:10.1016/j.berh.2018.08.005

16. Miao S, Xu T, Wu Y, et al. Extraction of BI-RADS findings from breast ultrasound reports in Chinese using deep learning approaches. Int J Med Inform. 2018;119:17-21. doi:10.1016/j.ijmedinf.2018. 08.009

17. Min L, Tian Q, Lu X, Duan H. Modeling EHR with the openEHR approach: an exploratory study in China. BMC Med Inform Decis Mak. 2018;18(1):75. doi:10.1186/s12911-018-0650-6

18. Li C, Xu X, Zhou G, et al. Implementation of National Health Informatization in China: survey about the status quo. JMIR Med Inform. 2019;7:e12238. doi:10.2196/12238

19. Shanghai Municipal Statistics Bureau. The age distribution of the household registration population in each district of Shanghai. Available from: http://tjj.sh.gov.cn/tjnj/nj19.htm?d1=2019tjnj/C0206. htm. Accessed September 8, 2020.

20. Boehme AK, Esenwa C, Elkind MS. Stroke risk factors, genetics, and prevention. Circ Res. 2017;120(3):472. doi:10.1161/CIRCRE SAHA. 116.308398

21. The China National Stroke Screening Survey. 2019. Available from: https://news.chinasdc.cn/NewsInfo/News/NewsListWeb?cCode= 00040003. Accessed September 8, 2020. 
22. Flegal KM, Kit BK, Orpana H, et al. Association of all-cause mortality with overweight and obesity using standard body mass index categories: a systematic review and meta-analysis. JAMA. 2013;309 (1):71-82. doi:10.1001/jama.2012.113905

23. Chinese Society of Health Management, Chinese Nutrition Society, Reproductive Medicine Branch of China International Exchange and Promotion Association for Medicine and Healthcare, China Health Promotion Foundation, Zhejiang Provincial Clinical Nutrition Center. Expert consensus and standard on weight management for overweight or obese people. Chin J Health Manage. 2018;12:200-207.

24. Mowery DL, Chapman BE, Conway M, et al. Extracting a stroke phenotype risk factor from Veteran Health Administration clinical reports: an information content analysis. $J$ Biomed Semantics. 2016;7:26. doi:10.1186/s13326-016-0065-1

25. Foraker RE, Shoben AB, Lopetegui MA, et al. Assessment of life's simple 7 in the primary care setting: the Stroke Prevention in Healthcare Delivery EnviRonmEnts (SPHERE) study. Contemp Clin Trials. 2014;38:182-189. doi:10.1016/j.cct.2014.03.007
26. Poppe KK, Doughty RN, Wells S, et al. Developing and validating a cardiovascular risk score for patients in the community with prior cardiovascular disease. HEART. 2017;103(12):891-892. doi:10.1136/ heartjnl-2016-310668

27. Liu M, Wu B, Wang W-Z, et al. Stroke in China: epidemiology, prevention, and management strategies. The Lancet Neurology. 2007;6(5):456-464. doi:10.1016/S1474-4422(07)70004-2

28. Xia X, Yue W, Chao B, et al. Prevalence and risk factors of stroke in the elderly in Northern China: data from the National Stroke Screening Survey. J Neurol. 2019;266(6):1449-1458. doi:10.1007/ s00415-019-09281-5

29. Li B, Wang T, Lou Y, et al. Sex differences in outcomes and associated risk factors after acute ischemic stroke in elderly patients: a prospective follow-up Study. J Stroke Cerebrovasc Dis. 2015;24 (10):2277-2284. doi:10.1016/j.jstrokecerebrovasdis.2015.06.007

30. Roquer J, Campello AR, Gomis M. Sex differences in first-ever acute stroke. Stroke. 2003;34(7):1581-1585. doi:10.1161/01.STR.000007 8562.82918.F6
Risk Management and Healthcare Policy

\section{Publish your work in this journal}

Risk Management and Healthcare Policy is an international, peerreviewed, open access journal focusing on all aspects of public health, policy, and preventative measures to promote good health and improve morbidity and mortality in the population. The journa welcomes submitted papers covering original research, basic science, clinical \& epidemiological studies, reviews and evaluations,

\section{Dovepress}

guidelines, expert opinion and commentary, case reports and extended reports. The manuscript management system is completely online and includes a very quick and fair peer-review system, which is all easy to use. Visit http://www.dovepress.com/testimonials.php to read real quotes from published authors. 